\title{
New AUA guideline tackles follow-up monitoring of small renal masses
}

A panel of expert urologists from across the USA, assembled by the AUA, has developed a set of guidelines for the follow-up of clinically localized renal cancer. These are the first AUA guidelines to deal specifically with the follow-up of a urological cancer. The panel's recommendations were presented at the AUA annual meeting in San Diego and have now been published in the Journal of Urology.

Follow-up monitoring of small renal masses is a complex issue, owing to the lack of parameters that can accurately determine which patients are likely to experience recurrence or progression. Moreover, long-term progression-free survival in these patients is high ( $>90 \%)$, leading to concerns of unnecessary follow-up procedures. Recent evidence revealed that a variety of imaging protocols exist without a consensus, leading to wide variations in radiation exposure. Thus, the panel were set the challenging task of achieving the fine balance of close disease monitoring without superfluous imaging procedures. "These guidelines represent the first step in standardizing the frequency and number of surveillance imaging studies to reduce variation and decrease costs after treatment for renal cancer," Marc Smaldone, who was not a member of the AUA panel, told Nature Reviews Urology.

The panel, led by Sherri Donat from Memorial Sloan-Kettering Cancer Center, performed a systematic review of the literature published between 1999 and 2011, based on key issues associated with follow-up monitoring (such as imaging and biopsy) of patients after the treatment for renal cancer, options for which range from active surveillance to ablative modalities and surgical resection. The final report contains 27 guideline statements based on the available evidence and expert opinion.

To limit radiation dose, the panel considered prudent use of CT and attempted to stratify patients into two groups - those at low risk (defined as pT1 with negative or unexamined lymph nodes) and those at moderate-to-high risk (defined as pT2-4 with or without positive lymph nodes) of recurrence or progression. For patients who have undergone surgery for low-risk localized renal cancer, the guidelines state that baseline postoperative CT or MRI should be performed within 3-12 months of partial nephrectomy. Thereafter, abdominal imaging with CT, MRI or ultrasonography should be performed annually for 3 years, although the panel are clear to state that more-frequent monitoring can be considered on the basis of individual risk factors.

Baseline postoperative imaging (CT or MRI) is recommended within 3-6 months of partial nephrectomy for patients with moderate-to-high-risk renal cancer, followed by imaging studies (CT, MRI, ultrasonography or chest radiography) every 6 months for at least 3 years, then annually for a further 2 years. PET imaging with labelled antibodies is not recommended owing to lack of data, although the panel recognize it's ongoing evaluation and potential role in the future.

Biopsy is another contentious issue in the monitoring of patients with small renal masses, especially those who choose to be managed with active surveillance. The AUA panel acknowledge that the accuracy of percutaneous renal biopsy has improved over the past few years and that tumour seeding seems to be an "exceedingly rare" event. Thus, the guidelines state that biopsy can be considered for patients commencing active surveillance. Imaging (CT or MRI) is recommended within 6 months of starting an active surveillance protocol, to establish a growth rate for the lesion.

Ablative procedures, such as radiofrequency ablation and cryoablation, are associated with higher recurrence rates

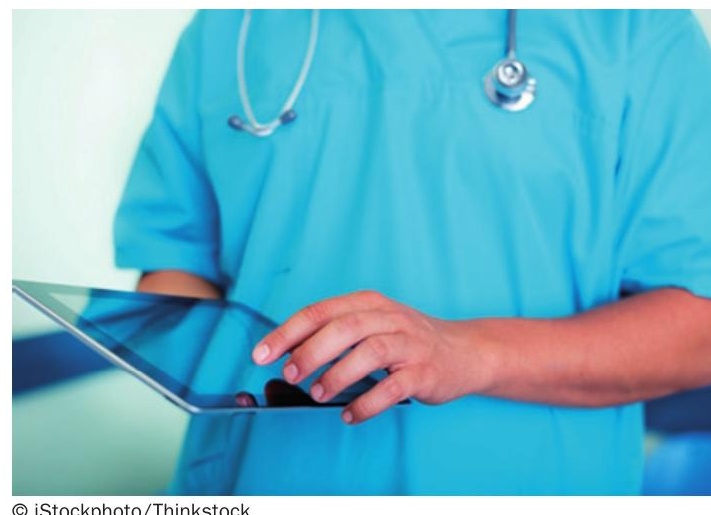

() iStockphoto/Thinkstock

than surgery. However, the published data on these new techniques are limited by the lack of criteria for ablation and definition of local recurrence, as well as insufficient follow-up duration. Accordingly, the panel recommend the use of pretreatment biopsy to guide the course of follow-up monitoring and the adoption of a standardized definition of treatment failure, based on the AUA guideline for management of the clinical T1 renal mass.

These guidelines are a welcome addition to the field, providing up-todate information to guide clinicians through the murky waters of follow-up monitoring of patients with clinically localized renal cancer. However, it must be noted that there remains a dearth of high-level evidence and many of the recommendations are based on expert opinion. "Post-treatment surveillance requires the careful balancing of risk and benefit," says Smaldone. "And even with the guidelines, this will often need to be tailored to the individual patient."

Sarah Payton

Original article Donat, M. et al. Follow-up for clinically localized renal neoplasms: AUA guideline. J. Urol. doi:10.1016/j.juro.2013.04.121

Further reading Almatar, A. \& Jewett, M. Time to standardize follow-up of low-stage renal cancer. Nat. Rev. Urol. 10, 257-259 (2013) 